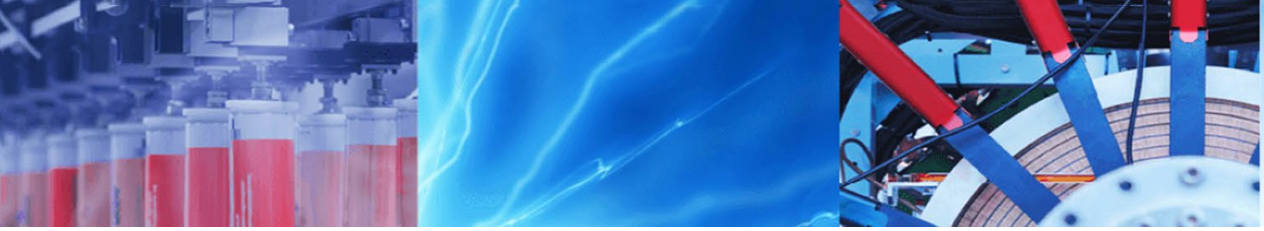

Research Article

\title{
Semi-analytical solution for some proportional delay differential equations
}

\author{
Mourad Chamekh ${ }^{1,2}$ (D) Tarig M. Elzaki ${ }^{1}$ Nabiha Brik $^{2}$
}

(c) Springer Nature Switzerland AG 2019

\begin{abstract}
In this work, we have developed a semi-analytical method, based on the combination of the differential transform method and the Laplace transform, to solve a class of linear and nonlinear differential equations with a proportional delay. In order to provide sufficient precision to meet computational needs, we have resumed some proofs of differential transform properties. Some illustrative and physical examples presented and compared to analytical solutions show promising results regarding the validity and applicability of this approach.
\end{abstract}

Keywords DDEs · Proportional delay · Differential transformation method · Multiple time delays

\section{Introduction}

Beyond the theoretical interest of study, the differential equations with proportional delay (DDEs) modeling by delays is necessary for many applied physical applications. An example in economics is the variations in a status at time $t$ as a function of the status of that time with some delay, which is inevitable in decision-making problems [1]. The DDEs have a wide range of applications in biology; the estimation of delay is a human orthostatic tremor [2], chemical kinetics [3] and fracture prediction under severe and multiaxial dynamic loading [4]. More generally, the DDEs is used by the domains under which some processes whose modeling is accompanied by a delayed dynamic part, where the evolution is determined from a value of the status $u(t)$ that depends on past values of the status, . In this case, it is necessary to consider a part of the "history" of the system in order to know its realistic evolution. For such needs, many researchers have been attracted by semi-analytic methods because the closed-form solution of DDEs is not always analytically accessible, or, having a method, but it is complicated, even if DDEs is linear. Therefore, some semi-analytical methods have been proposed such as the Adomian decomposition method [5], Elzaki transform method [6, 7], He's variational iteration method $[8,9]$ and the homotopy perturbation method [10].

The differential transformation method (DTM) has been introduced firstly by Zhou [11] to solve differential equations in electrical circuits domains. Since then, the DTM has been used to solve a large variety of equations. Relevant examples include integrodifferential systems, DDEs, differentialdifference equation, differential algebraic equation and onedimensional planar Bratu problem, [11]-[23]. In [18, 21], the authors have treated some problems using the DTM. In this work, we extend the use of this method for more generalized nonlinear differential equations and improve the convergence rate of DTM, and we have combined the Laplace transform (LT) and DTM to solve this type of equation. Using this proposed technique, we will show that there are a number of positive outcomes that can be achieved; among these are the greater flexibility and facility to overcome the problems of nonlinearity and difficulty of integration and the general delays, while respecting the conditions and features of the problem. In summation, we intend to treat some algebraic nonlinear DDEs of different degrees in the numerical examples.

We will consider the nonlinear differential equations type with a more time delays defined by

\footnotetext{
$\triangle$ Mourad Chamekh, mourad.chamekh@enit.utm.tn; Tarig M. Elzaki, Tarig.alzaki@gmail.com; Nabiha Brik, nabiha.brik@enit.utm.tn| ${ }^{1}$ College of Sciences and Arts, AlKamel, University of Jeddah, Jeddah, Kingdom of Saudi Arabia. ${ }^{2}$ National Engineering School at Tunis, LAMSIN, University of Tunis El Manar, Tunis, Tunisia.
} 
$u^{(p)}(t)=\varphi\left(t, u\left(\frac{t}{\alpha_{1}}\right), u\left(\frac{t}{\alpha_{2}}\right), \ldots, u\left(\frac{t}{\alpha_{n}}\right)\right)$,

where $\alpha_{i} \geq 1$ and $u^{(p)}$ are the $p$ th derivative of $u$ with respect to $t$, for $p, n \in \mathbb{N}$, and we suppose in this paper that $\varphi$ is a polynomial function. It will be easy to see that $\frac{t}{\alpha_{i}}=t-\tau_{i}$, with a delay given by $\tau_{i}=\left(1-\frac{1}{\alpha_{i}}\right) t$. Many traditional studies using DDEs consider only one time lag in their proposed models, but choosing two time lags or more time lags could really fit some needs of modeling and can be as close as possible to reality. For example, in [22] the authors have treated the problem HIV-1 infection with two time delays, where the same problem contains an intracellular delay and immune delay. In addition, we noted that these equations can also be classified into some types of higher-order neutral functional differential equations with proportional delay (NFDE). But the NFDE is a more general type of equation when the $p$ th derivative $u^{(p)}$ depends not only upon the history of $u$ but also on the history of $u^{(i)}$, where $i \leq p$.

\section{Differential transform method}

Following Zhou [11], we define the differential transform of a function $u$ by:

$U_{t_{0}}(i)=\frac{1}{i !}\left[\frac{\mathrm{d}^{i} u(t)}{\mathrm{d} t^{i}}\right]_{t=t_{0}}$,

and the inverse differential transform is defined by:

$u(t)=\sum_{i=0}^{\infty} U_{t_{0}}(i)\left(t-t_{0}\right)^{i}$.

The choice of this notation with the index $t_{0}$ is justified by the fact that the value of $U$ depends on $t_{0}$. The goal is to provide enough precision to meet calculation needs. This is precisely not to fall into the lack of precision committed by some authors (see the example [21]).

If $t_{0}=0$, we use the symbol $U$ that refers to the differential transform of $u$ without an index in the rest of this article (i.e., $U_{0} \equiv U$ ).

We summarize in Table 1 the main formulas of the monodimensional differential transform (see [23] for more details). We denote that $\delta$ is the Kronecker symbol and $p \in \mathbb{N}$.

\subsection{Application of DTM on the proportional delay}

In this section, we provide some necessary results proved in $[18,21]$, and these results can be used in the solution of Eq. (1) with proportional delay.
Table 1 Summarized formulas of the monodimensional DT

\begin{tabular}{ll}
\hline Functions & Differential transforms \\
\hline$h(t)=\alpha u(t)+\beta z(t)$ & $H_{t_{0}}(i)=\alpha U_{t_{0}}(i)+\beta Z_{t_{0}}(i)$ \\
$h(t)=\frac{\mathrm{d}}{\mathrm{d} t} u(t)$ & $H_{t_{0}}(i)=(i+1) U_{t_{0}}(i+1)$ \\
$h(t)=\frac{\mathrm{d}^{p}}{\mathrm{~d} t^{p}} u(t)$ & $H_{t_{0}}(i)=\frac{(i+p) !}{i !} U_{t_{0}}(i+p)$ \\
$h(t)=u(t) z(t)$ & $H_{t_{0}}(i)=\sum_{l=0}^{i} U_{t_{0}}(l) Z_{t_{0}}(i-l)$ \\
$h(t)=t^{p}$ & $H_{t_{0}}(i)=\delta(i-p)$ \\
\hline
\end{tabular}

Theorem 1 Let $U, F$ and $G$ be the differential transforms of $u$, fand $g$, respectively. For all $\alpha \in \mathbb{N}$, then

(1) If $u(t)=f\left(\frac{t}{\alpha}\right)$, then $U_{t_{0}}(i)=\frac{1}{\alpha^{i}} F_{t_{0}}(i)$.

(2) If $u(t)=\frac{\mathrm{d}^{p}}{\mathrm{~d} t^{p}} f\left(\frac{t}{\alpha}\right)$, then $U_{t_{0}}(i)=\frac{(i+p) !}{i !} \frac{1}{\alpha^{i+p}} F_{\frac{t_{0}}{\alpha}}(i+p)$.

(3) If $u(t)=f\left(\frac{t}{\alpha}\right) g\left(\frac{t}{\beta}\right)$, then $U_{t_{0}}(i)=\sum_{l=0}^{i} \frac{1}{\alpha^{\prime} \beta^{i-l}}$ $F_{\frac{t_{0}}{\alpha}}(I) G_{\frac{t_{0}}{\beta}}(i-l)$.

(4) If $u(t)=\frac{\mathrm{d}^{p}}{\mathrm{~d} t^{p}} f\left(\frac{t}{\alpha}\right) \frac{d^{q}}{d t^{q}} g\left(\frac{t}{\beta}\right)$, then

$$
U_{t_{0}}(i)=\sum_{l=0}^{i} \frac{(I+p) !(i-l+q)}{I !(i-l) ! \alpha^{l+p} \beta^{i-l+q}} F_{\frac{t_{0}}{\alpha}}(I+p) G_{\frac{t_{0}}{\beta}}(i-I+q) .
$$

Proof See "Appendix".

Remark 1 We note that in the special case when $t_{0}=0$ :

We obtain $\frac{t_{0}}{\alpha}=\frac{t_{0}}{\beta}=t_{0}=0$. Then, the differential transforms $U, F$ and $G$ are given in Theorem 1 without index.

\section{Description of the method}

Now consider Eq. (1) in the following short denotation

$u^{(p)}(t)=\varphi(t, u)$.

The first step is to take LT of both sides of the differential equation. The purpose is to seek some ways to relax the calculus by replacing the differentiation in the problem with an algebraic expression. The calculations cost reduction is clearly explained in the examples treated in this paper.

Taking the LT of both sides of Eq. (1), we obtain

$$
s^{p} \ell u(t)=\ell \varphi(t, u)+\sum_{i=1}^{p} s^{p-i} u^{i-1}(0),
$$

or 


$$
\ell u(t)=\frac{1}{s^{p}} \ell \varphi(t, u)+\sum_{i=1}^{p} \frac{1}{s^{i}} u^{i-1}(0) .
$$

Using the LT inverse, we have

$u(t)=\ell^{-1}\left\{\frac{1}{s^{p}} \ell \varphi(t, u)\right\}+\sum_{k=0}^{p-1} \frac{t^{k}}{k !} u^{k}(0)$.

In the second step, we apply the DTM which defines the solution $u(t)$ by the series

$u(t)=\sum_{i=0}^{\infty} U_{i}$

Substituting Eq. (5) into (4), we get the following algorithm $U_{0}=u(0)$,

$U_{1}=\sum_{k=1}^{p-1} \frac{t^{k}}{k !} u^{k}(0)$

$U_{i}=\ell^{-1}\left\{\frac{1}{s^{p}} \ell\left[A_{i}\right]\right\}, \quad i \geq 2$,

where $A_{i}$ is the differential transform of $\varphi(t, u)$. The DTM is efficient if $\varphi$ is a polynomial function in $u$. For more applicability, this method should be developed to cover more complex DDEs.

\section{Numerical examples}

In order to describe the technique of the DTM combined with the LT, we give some illustrative numerical examples.

Example 1 Consider the nonlinear DDEs

$\frac{\mathrm{d} u(t)}{\mathrm{d} t}=1-2 u^{2}\left(\frac{t}{2}\right), \quad t \in[0,1]$

$u(0)=0$,

The analytic solution is $u(t)=\sin t$.

Taking the LT of both sides of Eq. (9), to get;

$s \ell u(t)-u(0)=\frac{1}{s}-2 \ell\left[u^{2}\left(\frac{t}{2}\right)\right] \Rightarrow \ell u(t)=\frac{1}{s^{2}}-\frac{2}{s} \ell\left[u^{2}\left(\frac{t}{2}\right)\right]$.

Taking the inverse LT of Eq. (10), we get:

$u(t)=t-\ell^{-1}\left\{\frac{2}{s} \ell\left[u^{2}\left(\frac{t}{2}\right)\right]\right\}$.

Substituting Eq. (5) into (11), we have the following expression
$U(i+1)=-\ell^{-1}\left\{\frac{2}{s} \ell\left[A_{i}\right]\right\} \quad i \geq 0 \quad U(0)=0 \quad U(1)=t$.

where $A_{i}$ is the differential transform of $u^{2}\left(\frac{t}{2}\right)$ such that $A_{i}=\sum_{l=0}^{i} \frac{U(I) U(i-l)}{2^{\prime} 2^{i-l}}$.

Then,

$A_{1}=0 \Rightarrow U(2)=0$;

$A_{2}=\frac{t^{2}}{4} \Rightarrow U(3)=-\ell^{-1}\left[\frac{1}{s^{4}}\right]=-\frac{t^{3}}{3 !}$;

$A_{3}=0 \Rightarrow U(4)=0$;

$A_{4}=-\frac{t^{4}}{3 ! .8} \Rightarrow U(5)=-\ell^{-1}\left[-\frac{4 ! .2}{3 ! .8 s^{6}}\right]=\frac{t^{5}}{5 !}$;

and so on.

Then, the series solution according to Eq. (5) is given by

$u(t)=t-\frac{t^{3}}{3 !}+\frac{t^{5}}{5 !}-\frac{t^{7}}{7 !}+\cdots$

which is formally the same as Maclaurin series of $\sin t$.

Figure 1 shows the comparison between the exact solution and the approximated solution of the system (9). In Fig. 2, we remark that the proposed solution is characterized by two stages of precision: in the first stage, for $t \in[0,0.6]$ a well precise approximation of solution is obtained; in the second stage, the approximation is just reasonable $\left(<2 \times 10^{-4}\right)$.

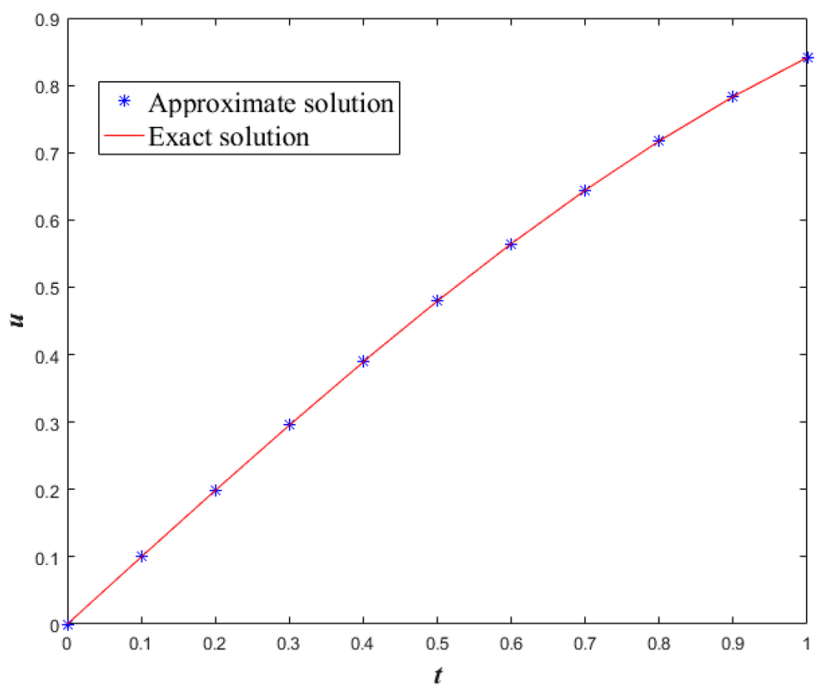

Fig. 1 The approximate solution is presented by blue stars and the analytical solution red solid line 


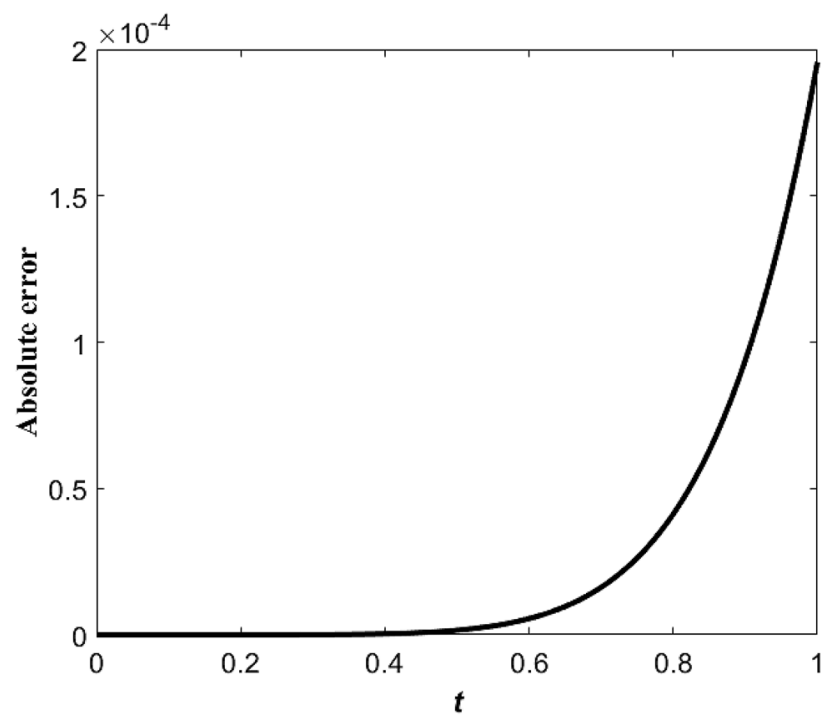

Fig. 2 Absolute error (Example 1).

Example 2 We consider here an example of DDEs with twotimes proportional delay,

$\frac{\mathrm{d} u(t)}{\mathrm{d} t}=u\left(\frac{t}{2}\right)-4 u\left(\frac{t}{4}\right), \quad t \in[0,1]$

$u(0)=1$.

The closed-form solution is $u(t)=\frac{3}{4} t^{2}-3 t+1$.

We apply the LT to Eq. (12a), and we get

$s \ell u(t)-u(0)=\ell\left(u\left(\frac{t}{2}\right)-4 u\left(\frac{t}{4}\right)\right)$.

By the inverse LT of Eq. (13), and using the initial conditions (12b), we obtain

$u(t)=t+\ell^{-1}\left[\frac{1}{s} \ell\left(A_{i}\right)\right]$.

Putting $A_{i}=\frac{U(i)}{2^{i}}-\frac{U(i)}{4^{i-1}}$, this is the differential transform of $u\left(\frac{t}{2}\right)-4 u\left(\frac{t}{4}\right)$.

Typically, we choose $U(0)=1$ and $U(1)=t$, but with this choice, the proposed technique fails to converge to the solution. The exact solution is given by $u_{\text {exat }}(t)=\frac{3}{4} t^{2}-3 t+1$. The trouble is we do not start by a consistent initial slope because $u_{\text {exat }}^{\prime}(0)=-3$ and the first-approximation $U(1)=t$. For this reason, we first propose to calculate the derivative $u^{\prime}(0)$ on the basis of Eq. (12a).
When $\mathrm{t}=0$, we have $u^{\prime}(0)=u(0)-4 u(0)=-3$ and we propose to put $U(1)=u^{\prime}(0) t=-3 t$. The objective is to ensure the consistency of initial slope.

$$
\begin{array}{rlrl}
U(i+1) & =\ell^{-1}\left\{\frac{1}{s} \ell\left[A_{i}\right]\right\}, & & i \geq 0 ; \\
U(0) & =1, U(1)=-3 t, & U(2)=\frac{3}{4} t^{2} .
\end{array}
$$

Then, the exact solution will be given by

$u(t)=U(0)+U(1)+U(2)=\frac{3}{4} t^{2}-3 t+1$.

Example 3 We consider the following nonlinear DDEs of the third order

$\frac{\mathrm{d}^{3} u(t)}{\mathrm{d} t^{3}}=-1+2 u^{2}\left(\frac{t}{2}\right), \quad t \in[0,1]$

$u(0)=0, \quad u^{\prime}(0)=1 \quad$ and $\quad u^{\prime \prime}(0)=0$.

The analytical solution is $u(t)=\sin t$.

Using the same methods as in Examples 1 and 2, we get the following relation

$U(i+2)=\ell^{-1}\left\{\frac{2}{s^{3}} \ell\left[A_{i}\right]\right\}, \quad i \geq 0, \quad U(0)=0$,

$U(1)=t, \quad U(2)=-\frac{t^{3}}{3 !}$,

where $A_{i}$ is the differential transform of $u^{2}\left(\frac{t}{2}\right)$ with $A_{i}=\sum_{l=0}^{i} \frac{U(I) U(i-l)}{2^{\prime} 2^{i-l}}$.

Then, we obtain

$A_{1}=0 \Rightarrow U(3)=0$;

$A_{2}=\frac{t^{2}}{4} \Rightarrow U(4)=\frac{t^{5}}{5 !}$;

$A_{3}=0 \Rightarrow(5)=0$;

and so on.

Hence, the series solution according to Eq. (5) will be given as

$u(t)=t-\frac{t^{3}}{3 !}+\frac{t^{5}}{5 !}-\frac{t^{7}}{7 !}+\cdots=\sin t$.

This example shows the performance of this method compared with the heavy calculations of Adomian's polynomials treated in [5] to solve the same example using Adomian decomposition method. Note further that by the 
inserting LT we overtake a few numbers of iterations in the calculation of solution compared with the DTM used in [21].

Example 4 (Hutchinson's equation) DDEs have been presented in several biological models. For example, we propose to study the logistic equation [24] or the Hutchinson's equation with a proportional delay for a single-species dynamics. This model is given by:

$\frac{\mathrm{d} u(t)}{\mathrm{d} t}=R u(t)-\frac{R}{K} u(t) u\left(\frac{t}{2}\right), \quad t \in[0,1]$

$u(0)=1$.

Consider $\mathrm{R}=1$ and $\mathrm{K}=1$. Applying the $\mathrm{LT}$ to both sides of Eq. (16a), we get

$$
\begin{aligned}
s \ell u(t)-u(0) & =\ell[u(t)]-\ell\left[u(t) u\left(\frac{t}{2}\right)\right] \\
\Rightarrow \ell u(t) & =\frac{1}{s-1}-\frac{1}{s-1} \ell\left[u(t) u\left(\frac{t}{2}\right)\right] .
\end{aligned}
$$

Taking the inverse LT of Eq. (17), we get:

$u(t)=\exp (t)-\ell^{-1}\left\{\frac{1}{s-1} \ell\left[u(t) u\left(\frac{t}{2}\right)\right]\right\}$.

Substituting Eq. (5) into (18), we have the following expression

$U(i+1)=-\ell^{-1}\left\{\frac{1}{s-1} \ell\left[A_{i}\right]\right\}, \quad i \geq 0$,

$U(0)=0, \quad U(1)=\exp (t)$,

where $A_{i}$ is the differential transform of $u(t) u\left(\frac{t}{2}\right)$ such that $A_{i}=\sum_{l=0}^{i} \frac{U(I) \cup(i-l)}{2^{i-l}}$.

Then,

$$
\begin{aligned}
A_{1} & =0 \Rightarrow U(2)=0 ; \\
A_{2} & =\frac{1}{2} \exp (2 t) \Rightarrow U(3)=-\frac{1}{2} \ell^{-1}\left[\frac{1}{(s-1)(s-2)}\right] \\
& =\frac{1}{2} \exp (t)-\frac{1}{2} \exp (2 t) ; \\
A_{3} & =0 \Rightarrow U(4)=0 ; \\
A_{4} & =\frac{5}{16}(\exp (2 t)-\exp (3 t)) \Rightarrow U(5) \\
& =-\frac{5}{16} \ell^{-1}\left[\frac{1}{(s-1)(s-2)}-\frac{1}{(s-1)(s-3)}\right] \\
& =\frac{5}{16} \ell^{-1}\left[\frac{1}{2(s-1)}-\frac{1}{(s-2)}+\frac{1}{2(s-3)}\right] \\
& =+\frac{5}{16}\left(\frac{1}{2} \exp (t)-\exp (2 t)+\frac{1}{2} \exp (3 t)\right) .
\end{aligned}
$$

Then, the approximate solution after five iterations is given by

$u(t)=\frac{63}{32} \exp (t)-\frac{23}{16} \exp (2 t)+\frac{15}{32} \exp (3 t) \cdots$

\section{Conclusion}

Even if the DDEs are linear, it is not easy to obtain an explicit analytical solution for these types of equations. For this purpose, we have developed a simple method for solving linear and nonlinear differential equations with proportional delay using a combination of the LT and the DTM. The treated examples emphasize that the proposed method is an effective tool to compute a semianalytical solution with a reasonable estimate. Besides that, the convergence rate can be improved by this combination of LT and DTM.

\section{Compliance with ethical standards}

Conflict of interest The authors declare that there are no conflicts of interest regarding the publication of this article.

Ethical approval This article does not contain any studies with human participants or animals performed by any of the authors

\section{Appendix}

Proof (Theorem 1)

(1) Calculating

$$
\frac{\mathrm{d}^{i}}{\mathrm{~d} t^{i}} u(t)=\left[\frac{\mathrm{d}^{i}}{\mathrm{~d} t^{i}} f\left(\frac{t}{\alpha}\right)\right]=\frac{1}{\alpha^{i}}\left[f^{(i)}\left(\frac{t}{\alpha}\right)\right] .
$$

Therefore $U_{t_{0}}(i)=\frac{1}{i !}\left[\frac{d^{i}}{d t^{i}} u(t)\right]_{t=t_{0}}=\frac{1}{i ! \alpha^{i}}\left[f^{(i)}\left(\frac{t}{\alpha}\right)\right]_{t=t_{0}}$ $=\frac{F_{\frac{t_{0}}{\alpha}}(i)}{\alpha^{i}}$.

Using the definition of DT, one gets $U_{t_{0}}(i)=\frac{1}{\alpha^{i}} F_{\frac{t_{0}}{\alpha}}(i)$.

(2) From (1), we have

$$
\begin{aligned}
U_{t_{0}}(i) & =\frac{1}{i !}\left[\frac{\mathrm{d}^{p}}{\mathrm{~d} t^{p}} u(t)\right]_{t=t_{0}}=\frac{1}{i ! \alpha^{i+q}}\left[f^{(i+q)}\left(\frac{t}{\alpha}\right)\right]_{t=t_{0}} \\
& =\frac{(i+q) !}{i ! \alpha^{i+q}} F_{\frac{t_{0}}{\alpha}}(i+q) .
\end{aligned}
$$


(3)

$$
U_{t_{0}}(i)=\frac{1}{i !}\left[\frac{\mathrm{d}^{i}}{\mathrm{~d} t^{i}} u(t)\right]_{t=t_{0}}=\frac{1}{i !}\left[\frac{\mathrm{d}^{i}}{\mathrm{~d} t^{i}}\left(f\left(\frac{t}{\alpha}\right) g\left(\frac{t}{b}\right)\right)\right]_{t=t_{0}} .
$$

Using the Leibnitz's theorem we get

$=\sum_{l=0}^{i}\left(\begin{array}{l}i \\ l\end{array}\right) \frac{(i-l) ! l !}{i !} \frac{1}{\alpha^{\prime}} \frac{1}{l !}\left[f^{(l)}\left(\frac{t}{\alpha}\right)\right]_{t=t_{0}} \frac{1}{b^{i-l}} \frac{1}{(i-l) !}\left[g^{(i-l)}\left(\frac{t}{b}\right)\right]_{t=t_{0}}$.

$=\sum_{l=0}^{i} \frac{1}{\alpha^{l} \beta^{i-l}} F_{\frac{t_{0}}{\alpha}}(I) G_{\frac{t_{0}}{\beta}}(i-l)$.

(4) We have

$U_{t_{0}}(i)=\frac{1}{i !}\left[\frac{\mathrm{d}^{i}}{\mathrm{~d} t^{i}} u(t)\right]_{t=t_{0}}=\frac{1}{i !}\left[\frac{\mathrm{d}^{i}}{\mathrm{~d} t^{i}}\left(\frac{\mathrm{d}^{p}}{\mathrm{~d} t^{p}} f\left(\frac{t}{\alpha}\right) \frac{\mathrm{d}^{q}}{\mathrm{~d} t^{q}} g\left(\frac{t}{\beta}\right)\right)\right]_{t=t_{0}}$.

From the Leibnitz's theorem of ith-derivative of a product we get

$$
\begin{aligned}
& =\frac{1}{i !} \sum_{l=0}^{i}\left(\begin{array}{l}
i \\
l
\end{array}\right) \frac{1}{\alpha^{l+p}}\left[f^{(I+p)}\left(\frac{t}{\alpha}\right)\right]_{t=t_{0}} \frac{1}{\beta^{i-l+q}}\left[g^{(i-l+q)}\left(\frac{t}{\beta}\right)\right]_{t=t_{0}} \\
& =\frac{1}{i !} \sum_{l=0}^{i}\left(\begin{array}{l}
i \\
l
\end{array}\right)\left(\frac{(I+p) ! F_{\frac{t_{0}}{\alpha}}(I+p)}{\alpha^{I+q}}\right)\left(\frac{(i-I+q) ! G_{\frac{t_{0}}{\beta}}(i-I+q)}{b^{i-l+q}}\right) \\
& =\sum_{l=0}^{i} \frac{(I+p) !(i-I+q) !}{I !(i-l) ! \alpha^{l+p} \beta^{i-l+q}} F_{\frac{t_{0}}{\alpha}}(I+p) G_{\frac{t_{0}}{\beta}}(i-I+q) .
\end{aligned}
$$

\section{References}

1. Bayraktar E, Egami M (2007) The effects of implementation delay on decision-making under uncertainty. Stoch Process Appl 117:333-358

2. Müller T, Lauk M, Reinhard et al (2003) Estimation of delay times in biological systems. Ann Biomed Eng 31(11):1423-1439

3. Roussel MR (1996) The use of delay differential equations in chemical kinetics. J Phys Chem 100(20):8323-8330

4. Allix O, Deü J-F (1997) Delayed-damage modelling for fracture prediction of laminated composites under dynamic loading. Eng Trans 45(1):29-46

5. Evans DJ, Raslan KR (2005) The Adomian decomposition method for solving delay differential equation. Int J Comput Math 82(1):49-54
6. Alderremy AA, Elzaki TM, Chamekh M (2018) New transform iterative method for solving some Klein-Gordon equations. Results Phys 10:655-659

7. Elzaki TM, Chamekh M (2018) Solving nonlinear fractional differential equations using a new decomposition method. Univ J Appl Math Comput 6:27-35

8. He JH (1997) Variational iteration method for delay differential equations. Commun Nonlinear Sci Numer Simul 2:235-236

9. Chamekh M, Elzaki TM (2018) Explicit solution for some generalized fluids in laminar flow with slip boundary conditions. J Math Comput Sci 18:272-281

10. Shakeri F, Dehghan M (2008) Solution of delay differential equations via a homotopy perturbation method Math. Comput Model 48:486-498

11. Zhou JK (1986) Differential transformation and its application for electrical circuit. Huazhong University Press, Wuuhahn (in Chinese)

12. Abdel-Halim IH, Ertrk V (2007) Applying differential transformation method to the one dimensional planar Bratu problem. Int J Contemp Math Sci 2(30):1493-1504

13. Arikoglu A, Ozko I (2006) Solution of differential-difference equations by using differential transform method. Int J Comput Math 181(1):153-162

14. Arikoglu A, Ozko I (2008) Solution of integral and integrodifferential systems by using differential transform method. Int J Comput Math 56:2411-2417

15. Thongmoon M, Pusjuso S (2010) The numerical solutions of differential transform method and the Laplace transform method for a system of differential equations. Nonlinear Anal Hybrid Syst 4:425-431

16. Fatoorehchi $\mathrm{H}$, Abolghasemi $\mathrm{H}$, Magesh N (2015) The differential transform method as a new computational tool for Laplace transforms. Natl Acad Sci Lett 38(2):157-160

17. Ayaz F (2004) Applications of differential transform method to differential-algebraic equations. Appl Math Comput 152:649-657

18. Karakoç F, Bereketoğlu H (2009) Solution of delay differential equation by using differential transform. Int J Comput Math 86(5):1-6

19. Kurnaz A, Oturanç $G$ (2005) The differential transform approximation for the system of ordinary differential equations. Int J Comput Math 82(6):709-719

20. Liu H, Song Y (2007) Differential transform method applied to high index differential-algebraic equations. Appl Math Comput 184(2):748-753

21. Šmarda Z, Diblík J, Khan Y (2013) Extension of the differential transformation method to nonlinear differential and integrodifferential equations with proportional delays. Adv Differ Equ 2013:69

22. Pawelek KA, Liu S, Pahlevani F, Rong L (2012) A model of HIV-1 infection with two time delays: mathematical analysis and comparison with patient data. Math Biosci 235:98-109

23. Elzaki TM (2012) A solution of nonlinear differential equations using mixture of Elzaki transform and transform method. Int Math Forum 7(13):631-638

24. Ruan S (2006) Delay differential equations and applications. Springer, Berlin, pp 477-517 